\title{
¿Salimos a comer? Un análisis de la relación entre condiciones socioeconómicas y consumo de alimentos fuera del hogar en Colombia, 1993-2014
}

\author{
Giselle Torres-Pabón \\ Universidad Santiago de Cali. Facultad de Ciencias Económicas y Empresariales \\ gtorres1@uc.cl \\ ORCID: https://orcid.org/0000-0002-1602-8249
}

\section{Resumen}

El objetivo de este artículo es analizar el gasto en consumo de alimentos fuera de casa en función de las condiciones socioeconómicas de los hogares colombianos entre 1993 y 2014. La metodología es cuantitativa, es decir, se trata de un análisis descriptivo y modelos de regresión lineal. La variable dependiente es la proporción del gasto en consumo de alimentos fuera del hogar, y las variables independientes son la presencia de al menos una mujer en el hogar, la edad del jefe del hogar, los niveles educativos, la calidad de vida, el sector y la región. Los datos utilizados provienen de la Encuesta Nacional de Calidad de Vida, realizada por el Departamento Nacional de Estadística colombiano. Las hipótesis son las siguientes: (1) comer fuera del hogar se ha incrementado y (2) el consumo de alimentos aumenta en las zonas urbanas, en los hogares de altos niveles educativos, con altas condiciones de vida y sin presencia de mujeres en el hogar. Los resultados han confirmado las hipótesis propuestas. Analizando los resultados en el contexto estudiado, podemos afirmar que existe diversificación y diferenciación en la práctica alimentaria colombiana, específicamente en el consumo de alimentos fuera del hogar. Además, se comprueba y se halla un patrón alimentario del consumo de alimentos fuera del hogar en diferentes latitudes, lo que llama a la reflexión, en la sociología de la alimentación, a seguir indagando y problematizando las metodologías y las aproximaciones teóricas y empíricas que tenemos para aproximarnos a la comprensión de la realidad por medio del estudio del consumo de alimentos.

Palabras clave: consumo de alimentos fuera del hogar; presupuesto del hogar; servicio alimentario; condiciones socioeconómicas; estratificación social; diversificación alimentaria; Colombia 
Abstract. Do we eat-out? Socioeconomic conditions and eating-out in Colombia, 1993-2014

This article aims to analyze the association between expenditure on eating-out and households' socioeconomic conditions in Colombian (1993-2014). The methodology is quantitative: a descriptive analysis and linear regression models. The dependent variable is the proportion of expenditure on eating-out; and the independent variables are: at least one woman in the household, age of the head of the household, educational levels, floor material, sector and region. The data is the National Quality of Life Survey, carried out by the Colombian National Department of Statistics. The hypotheses are: (1) Eating outside the home has increased and (2) food consumption increases in urban areas, in homes with high educational levels, with high living conditions and without the presence of women in the home. The results have verified the proposed hypotheses. Analyzing the results in context, they allow to affirm the existence of diversification and differentiation in Colombian food practice, specifically in what implies eating-out. In addition, a dietary pattern of eating-out is verified and found in different latitudes, which calls for reflection, in the Sociology of Food Consumption, to continue investigating and problematizing the methodologies and theoretical and empirical approaches that we have to approach the understanding of reality through the study of food consumption.

Keywords: eating-out; household budget; foodservice; socioeconomic conditions; social stratification; food diversification; Colombia

\section{Sumario}

\section{Introducción}

2. Revisión de la literatura

3. Contexto del sector servicio de alimentos en América Latina y Colombia

4. Datos y metodología

5. Resultados
6. Discusión

7. Conclusiones

Agradecimientos

Financiación

Referencias bibliográficas

\section{Introducción}

En el mundo occidental, consumir alimentos bajo el único gran formato - permitido y bien visto- de sentarse a la mesa con todas las piezas posibles de la etiqueta (cuchillos, tenedores, cucharas, servilletas, etcétera), con los productos obtenidos y preparados bajo determinadas normas rigurosas y con un específico comportamiento, como ejemplo de urbanismo, es cosa del pasado (Elias, 2016; Neirinck y Poulain, 2001).

En diferentes latitudes, los modos de ejercer la práctica ${ }^{1}$ alimentaria han cambiado, junto con las transformaciones de las estructuras culturales, políticas

1. «A practice (Praktik) is a routinized type of behavior which consists of several elements, interconnected to one other: forms of bodily activities, forms of mental activities, things and their use, a background knowledge in the form of understanding, know-how, states of emotion and motivational knowledge» or «a routinized way in which bodies are moved, objects are handled, subjects are treated, things are described and the world is understood" (Reckwitz, 2002: 249 y 250). 
y económicas de cada espacio y en cada tiempo. Ya no comemos lo mismo, ni de la misma manera, que veinte años atrás, y cada vez son más las cantidades y los diversos bienes y servicios alimenticios disponibles en menor tiempo (Fischler, 1979). La diversificación y la distinción —en imaginarios, discursos, representaciones, prácticas, sentires y materialidades — son, en conjunto, una realidad (Edwards, 2013; Warde et al., 1999), y en la literatura a estos cambios se los ha nominado modernidad alimentaria (Fischler, 1979; Grignon y Grignon, 1999). Sin embargo, hay continuidades y la fuerza de la tradición se mantiene en otros elementos que configuran la práctica alimentaria (Poulain, 2002; Régnier et al., 2006; Warde, 1997).

Las rupturas y continuidades hacen explícitos los conflictos y consensos entre agencias y estructuras; entre imaginarios, discursos, representaciones y materialidades; entre posibilidades y limitaciones, o entre lo tradicional y lo moderno que compone la práctica alimentaria (bienes, servicios, modales, etcétera). Con esto en mente, el objetivo de este artículo es analizar la relación entre el gasto en consumo de alimentos fuera de los hogares colombianos y sus condiciones socioeconómicas para los años 1993, 1997, 2010 y 2014. La pregunta para responder es la siguiente: ¿cómo las condiciones socioeconómicas moldean el consumo de alimentos fuera del hogar en Colombia entre 1993 y 2014? Las hipótesis que guían el artículo son estas: (1) el consumo de alimentos fuera del hogar se incrementó en el periodo analizado, y (2) el consumo de alimentos aumenta en las zonas urbanas, en los hogares de altos niveles educativos, con altas condiciones de vida y sin presencia de mujeres en el hogar.

La metodología es cuantitativa, es decir, se trata de un análisis descriptivo y modelos de regresión lineal. La variable dependiente es la proporción del gasto en consumo de alimentos fuera del hogar, y las variables independientes son la presencia de al menos una mujer en el hogar, la edad del jefe del hogar, los niveles educativos, la calidad de vida, el sector (urbano/rural) y la región. Los datos utilizados provienen de la Encuesta Nacional de Calidad de Vida, realizada por el Departamento Nacional de Estadística colombiano.

\section{Revisión de la literatura}

Una ruptura en la práctica alimentaria está en el incremento del consumo de alimentos fuera del hogar, modalidad que compite con el consumo de alimentos en el hogar, que representa la tradición par excellence (Díaz-Méndez y Brock, 2017; Elias, 2016; Euromonitor International, 2018a; Neirinck y Poulain, 2001; Poulain, 2002). La categoría comer fuera del hogar puede ser entendida a partir de diferentes tipologías: comida callejera, comida en el lugar de trabajo, comida en restaurantes, cafeterías, cines u otro tipo de lugares en los que comer implique el pago por un servicio. A su vez, comer fuera del hogar puede implicar también comer en otros hogares, ya sea de familiares o amigos, o en el bus o al caminar o, en general, en un lugar distinto al de residencia del hogar, ya sea un parque, un paseo de río, de mar, entre otros, que no involucre una transacción comercial o monetaria (Kocevski y Risteski, 2012; Larmet, 2002). 
Así pues, dado que son múltiples las interpretaciones que pueden darse en torno a esta modalidad de consumo alimentario, se propone como punto de partida la conceptualización realizada por Warde y Martens (2000), quienes, en el texto Eating out: Social diferentiation, consumption and pleasure, afirman que existen al menos seis elementos en las percepciones que definen aquello que se llama comer fuera del hogar (eating out), en contraste con comer en los hogares. El primer aspecto es una diferenciación espacial: no es el hogar. El segundo aspecto está asociado al trabajo que requiere la preparación de los alimentos. Los alimentos han sido preparados por personas diferentes al comensal y los otros comensales que componen el hogar. El tercer aspecto se refiere a una transacción, ya que existe un pago, es una actividad comercial. El cuarto elemento es su carácter social. Comer fuera del hogar está asociado a una actividad social, es decir, a la idea de compartir con amigos, familiares o incluso con otras personas presentes en el lugar, con quienes se comparte otro tipo de vínculos. El quinto aspecto es el sentido de la acción, pues se trata de un evento especial. Y el sexto y último criterio es la estructura de la ingesta: tiempos y composición, lo que remite a dudas sobre qué se come, cuántas cantidades se ingieren, de cuánto tiempo se dispone para desarrollar la acción, etcétera.

De modo que la categoría comer fuera del hogar es una actividad específica socioespacial, que envuelve una provisión comercial, que es hecha por otra persona, en una ocasión especial, que tiene múltiples razones para ser ejecutada de esa manera: placer, ocio o necesidad (Warde y Martens, 2000). A su vez, los autores tienen presente la evolución de las posibilidades de aprovisionamiento de alimentos bajo esta modalidad, enmarcada en un ciclo en producciónconsumo (Warde y Martens, 2000).

Entonces, bajo esta teorización, es posible afirmar que en la categoría comer fuera del hogar se encuentran perspectivas de la sociología de la alimentación y de la sociología del mercado que conjugan diferentes elementos, dinámicos en el tiempo y en el espacio, correspondientes a las condiciones socioeconómicas, estratificadas, materiales o no materiales, de nivel micro o macro, que constriñen o posibilitan el desarrollo de dicha actividad (Desjeux, 2006; DubuissonQuellier, 2016; Dubuisson-Quellier y Plessz, 2013; Herpin, 2004; Larmet, 2002; Le Velly, 2012; Warde y Martens, 2000).

En otras palabras, comer fuera del hogar, en un lugar comercial, pone de manifiesto la existencia de un conjunto de agencias y estructuras que están en consenso o en conflicto. Esto significa que comer fuera del hogar puede ser una decisión, una preferencia o una necesidad, una rutina o un hábito, ya sea por motivos laborales, educativos, etarios, de salud, de celebración, de ingresos, de género, etcétera, dadas las posibilidades o limitaciones de acceso a los servicios alimentarios (precios, lugares, variedad, calidad, entre otros) en un espacio y tiempo determinados (Arango et al., 2010; Cabiedes-Miragaya, 2017; Fischler, 1979; Warde y Martens, 2000).

Por otra parte, entre las posibilidades (o limitaciones) propias de los hogares, existen evidencias que corroboran la asociación negativa entre la presencia de mujeres en el hogar y el consumo de alimentos fuera del hogar (Martens, 
1997; Lund et al., 2017; Wood, 1992), y la incidencia positiva del sector urbano, el nivel de ingresos y el nivel educativo en este tipo de consumo (Martens, 1997; Lund et al., 2017).

De modo que un análisis complejo, que integre aspectos sociales y económicos para la categoría comer fuera del hogar, ya sean agenciales o estructurales, implica reconocer que existen diferentes elementos: tipos de ingestas (comidas o snacks), el tiempo que se emplea para desarrollarlas, las motivaciones, las personas que ejecutan y con quienes se comparte dicho momento, el presupuesto destinado para este tipo de consumo alimentario, los lugares donde se desarrolla la acción, las características de dichos lugares, las características del sector servicio alimentario, las políticas públicas, etcétera. Y las características agenciales de los hogares son varias: género, nivel educativo, edad, ocupación, entre otras. Aunque esta complejidad debe ser clara para el lector, este artículo se enfoca en el análisis de la relación entre las condiciones socioeconómicas de los hogares y el gasto destinado al consumo fuera del hogar.

Este trabajo busca contribuir al análisis de los patrones de gasto del consumo de alimentos fuera del hogar, en un contexto diferente a los ya estudiados. A su vez, pretende ser un aporte al análisis de los cambios en las prácticas alimentarias, bajo un contexto latinoamericano, cuyas transformaciones en términos de instituciones o reglas de juego y estructuras de comercialización de bienes y servicios han variado durante el periodo analizado. Además, estas son distintas a las de los países europeos o industrializados, como los Estados Unidos o el Canadá, lugares donde mayoritariamente se ha producido esta literatura. Por último, este texto busca mostrar cómo las particularidades del sector servicio de alimentos, de acuerdo con un contexto socioeconómico específico, afecta los modos en que se desarrolla la práctica alimentaria, bajo la modalidad de consumo de alimentos fuera del hogar.

Ahora bien, con las fuentes disponibles en Colombia, no es posible hacer un seguimiento de los consumidores de alimentos fuera de los hogares en términos de las motivaciones, las personas con quienes comparten sus alimentos, el tiempo que destinan al consumo de los alimentos, los lugares que frecuentan, los precios que pagan, entre otras características intrínsecas al consumo de alimentos. A pesar de esta limitación, para comprender los resultados obtenidos con relación a la proporción del gasto en alimentos fuera del hogar, este artículo considera de absoluta importancia el contexto en el que se desarrolla esta investigación (Barbosa et al., 2018; Maloutas y Fujita, 2012; Díaz-Méndez y García-Espejo, 2017; Prättälä et al., 1992), y las inferencias que se pueden hacer a partir del texto son absolutamente valiosas para comprender los resultados que se presentarán. Por lo tanto, la siguiente sección tiene como objetivo presentar al lector el contexto donde tiene lugar la práctica alimentaria, específicamente en lo que corresponde al consumo de alimentos fuera de los hogares. Por lo tanto, la estructura de este artículo es presentar primero las características del sector servicio de alimentos en América Latina y en Colombia. Seguidamente, se expondrán los datos y la metodología empleada (análisis descriptivo y análisis de regresión lineal). En tercer lugar, se compartirán los resultados 
obtenidos y, finalmente, se discutirán dichos resultados y se presentarán las conclusiones.

\section{Contexto del sector servicio de alimentos en América Latina y Colombia}

En América Latina el sector servicio de alimentos ha crecido y se ha diversificado de manera continua en el periodo de análisis de este trabajo. Los hogares tienen más opciones y más variadas para ejecutar consumos de alimentos fuera del hogar (Euromonitor International, 2015; 2018b; 2018d). Además, en América Latina — como región altamente diversa - el sector está localizado y adaptado a los conceptos y cocinas específicas de cada país latinoamericano, a los consumidores y a sus niveles de ingresos.

Sumado a lo anterior, el cambio en las ciudades latinoamericanas, sus sistemas y redes de transportes y las modificaciones en las jornadas laborales hacen que, cada vez más, haya una mayor predisposición a hacer uso de los diversos servicios alimentarios existentes en los diferentes países de la región, y con el apoyo de las políticas públicas dirigidas a regular el mercado alimentario. De modo que estamos hablando de una modalidad en la práctica alimentaria que conjuga las características propias de los agentes, del sistema de aprovisionamiento, del contexto, de las estructuras citadinas y de sus políticas públicas.

Específicamente Colombia, partir de la Constitución política de 1991, ha transitado por un largo y difícil camino de reestructuración social, política y económica. Aunque la Constitución nació con el objetivo de buscar la paz y la amplitud de derechos políticos, económicos y ciudadanos de los colombianos, los resultados han sido múltiples en materia fiscal, política, mercantil y social (Perry, 2011). Por eso, Colombia es un país que se debate entre lo tradicional y lo moderno; entre productos y servicios locales o internacionales; entre estructuras citadinas y rurales; entre la apertura económica y el interés de fortalecer al agro y la industria nacional. Al mismo tiempo, vive la pobreza y la violencia estructural, un largo conflicto interno entre diferentes grupos armados (legales y no legales) y la desigualdad en múltiples esferas de la vida cotidiana.

En el día a día, un aspecto lleno de contradicciones es la práctica alimentaria. Es posible decir que en Colombia la práctica alimentaria se está redefiniendo, por las nuevas ofertas comerciales ${ }^{2}$ (lugares y productos), las nuevas estructuras industriales y citadinas, las nuevas demandas de los consumidores y los nuevos discursos e imaginarios alimentarios, entre otros. En la práctica alimentaria, específicamente en lo concerniente al consumo de alimentos fuera

2. Los datos disponibles de Euromonitor son de 2012 a 2017. El análisis de este documento abarca el periodo 1993-2014. Con la información disponible, es posible decir que durante el periodo de análisis, al menos de 2012 a 2014, las unidades de alimentos consumidos fuera del hogar se incrementaron un 5,48 \% (de 59,085 a 62,322); y a precios constantes el valor de ventas se incrementó un 7,33 \% (de 27,243 a 29,240 billones de pesos a precios constantes) (Euromonitor International, 2018b). Esta información busca mostrar al lector que existen pruebas para afirmar que el mercado del sector servicio de alimentos aumentó en Colombia en el periodo analizado. 
del hogar, la economía colombiana tiene un alto porcentaje de informalidad ${ }^{3}$ en el sector servicio de alimentos, paralelo al incremento de oferentes y puntos de venta de servicios de cadenas ${ }^{4}$ alimenticias nacionales e internacionales, cuyas diversas y amplias estrategias buscan incidir en los consumidores colombianos y sus hábitos alimenticios. Además, el entramado institucional se enfoca en defender el llamado espacio público y los estándares de salubridad alimentaria, de modo que estamos frente a un sistema de aprovisionamiento que está cambiando, desde hace varios años y de manera sostenida, en términos de oferentes, consumidores y reglas de juego (Euromonitor International, 2018b; 2018c; 2018d).

El sector servicio de alimentos en Colombia se compone de diferentes modalidades: para llevar o delivery, café/bar, ${ }^{5}$ restaurantes, ${ }^{6}$ comidas rápidas, ${ }^{7}$ cafeterías autoservicio, comidas callejeras/quioscos. ${ }^{8}$ Se trata de un sector que depende mucho de las condiciones socioeconómicas de un país o región. Al comparar el sector entre países emergentes o desarrollados, se puede afirmar que cada modalidad toma diferente predominancia en el uso, ya sea por elementos culturales, por facilidades en precios y niveles de ingresos, por las posibilidades de acceso a lugares, entre otros. Es decir, la modalidad de consumo de alimentos fuera del hogar depende mucho también del sistema de aprovisionamiento de un país en un momento determinado (Euromonitor

3. Puestos callejeros de fritangas (fritos: empanadas, papas rellenas, dedos de queso, buñuelos), comidas rápidas (hamburguesas, hot-dogs, pizzas) y otros.

4. Crepes \& Waffles SA, Frisby SA, McDonald's Corp, Sandwich Qbano SA, Domino’s Pizza Inc, Papa John's, entre otros.

5. «All establishments where the focus is on drinking (either alcoholic or non-alcoholic beverages). While a wide variety of snacks and full meals are offered, it is not uncommon for consumers to only order a drink. As a general rule, establishments deriving $50 \%$ of their income or more from the sale of drinks are included here» (Euromonitor, 2018a: 44)

6. "All sit-down establishments where the focus is on food rather than on drink. Menus offer multiple selections, and may include breakfast, lunch and dinner. Preparation of food products is often complex and involves multiple steps» (Euromonitor, 2018a: 44).

7. «Outlets offer limited menus that are prepared quickly. Customers order, pay and pick up their order from a counter. Outlets tend to specialize in one or two main entrées such as hamburgers, pizza, ice cream or chicken, but they usually also provide salads, drinks, dessert etc. Food preparation is generally simple and involves one or two steps, allowing for kitchen staff generally consisting of younger, unskilled workers. Other key characteristics include: • A standardized and restricted menu; $\bullet$ Food for immediate consumption; $\bullet$ Tight individual portion control on all ingredients and on the finished product; $\bullet$ Individual packaging of each item; $\bullet$ Counter service; $\bullet$ A seating area, or close access to a shared seating area, such as in a shopping center food court $\bullet$ For chained fast food, chained and franchised operations which operate under a uniform fascia and corporate identity. $\bullet$ Take out is generally present, as is drive-through in some markets» (Euromonitor, 2018a: 45).

8. «Small, sometimes mobile, foodservice providers characterised by a limited product offering and by low prices. Includes street stalls, street hawkers and foodservice kiosks where food is prepared in some way and served through a hatch or over a display counter to take away. Also includes kiosks and carts located externally or internally shopping malls, etc. As a rule, street stalls/kiosks outlets tend to be smaller than $100 \%$ home delivery/takeaway outlets, while menus are more limited, often (though certainly not always) with a greater emphasis on snack items, rather than full meals» (Euromonitor, 2018a: 46). 
Figura 1. Empresas que ofertan bienes alimenticios (unidades en miles y por año)

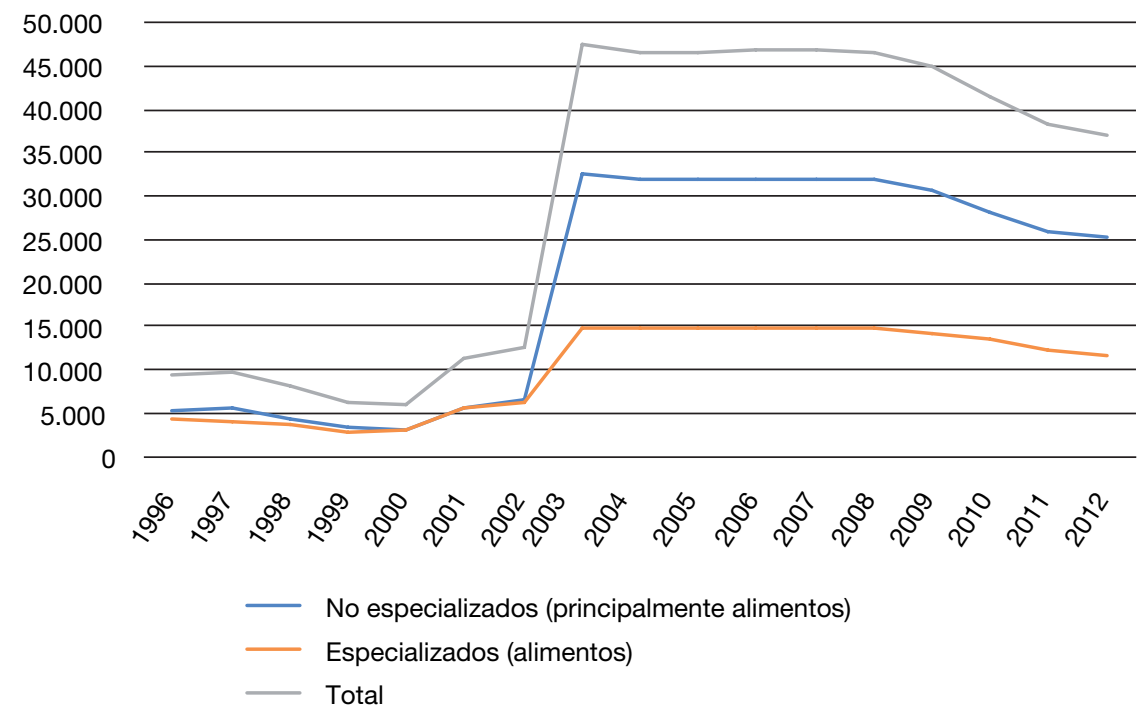

Fuente: elaboración propia, a partir de la encuesta EAC-DANE.

International, 2018a). En línea con la Encuesta Anual de Comercio (ANC), realizada por el Departamento Nacional de Estadística colombiano (DANE), los lugares que ofertan bienes alimenticios se incrementaron en el tiempo de análisis (figura 1).

Como se dijo anteriormente, el lector debe ser consciente de que, con la información disponible por el momento, en muchos países de América Latina, y también en Colombia, no es posible adentrarse en la profundidad de las relaciones entre consumidores y el mercado de servicios alimentarios, preocupaciones de la sociología de la alimentación y de la sociología del mercado. Sin embargo, presentar un breve panorama del sector tiene como objetivo señalar que no se debe perder de vista que la práctica alimentaria conjuga condiciones materiales o no materiales, de niveles micro o macro, que corresponden a determinados lugares y tiempos. Aunque el foco de este artículo es el análisis de la relación entre las condiciones económicas de los hogares y la proporción del gasto que estos destinan al consumo de alimentos fuera del hogar, para esta investigación se considera relevante no dejar de nombrar las transformaciones que vive Colombia en este aspecto, lo que también permite hacer inferencias sobre lo que ocurre en la práctica alimentaria en el tiempo y el espacio en el que tiene lugar esta investigación.

Ahora bien, respecto al estudio del consumo de alimentos fuera del hogar en América Latina, se pueden citar algunos artículos relevantes. En México, es el caso de Llamas et al. (2012), bajo un modelo logit binomial, cuya variable dependiente es dicotómica (1: si gasta / 0: no gasta en alimentos y bebidas 
fuera del hogar), y sus variables independientes son las siguientes: nivel de ingreso del hogar, jefe del hogar es mujer, edad del jefe del hogar, nivel educativo del hogar, cantidad de personas en el hogar, presencia de menores de 12 años, presencia de mayores de 65 años, becarios, ocupados, horas trabajadas semanalmente por los miembros del hogar, zona rural o urbana del hogar. Los autores hallaron que las variables ingreso, nivel educativo y localidad urbana tienen un sentido positivo con la variable dependiente, mientras que las variables mujer, edad, menores de 12 y mayores de 65 tienen un sentido negativo con la variable dependiente.

Por otra parte, García Arancibia et al. (2013) se refieren al caso argentino, también bajo un modelo logit binomial, donde la variable dependiente es la siguiente: 1: si hay consumo de alimentos fuera del hogar; 0: no hay. Las variables independientes son las siguientes: jefe mujer, edad del jefe del hogar, cantidad de miembros del hogar, menores de 14, mayores de 65, si en el hogar predominan hombres, tipo de hogar, log del gasto total. Los autores reportan que existe una relación positiva con la probabilidad de consumir alimentos fuera del hogar de las variables hogares unipersonales y hogares donde predominan los hombres, y una relación negativa con las variables cantidad de personas en el hogar, menores de 12, mayores de 65 y hogares con jefe mujer.

En Brasil, Nogueira et al. (2013) estudiaron la frecuencia de individuos que consumieron alimentos fuera del hogar respecto a la edad del jefe del hogar, el sexo, el área de localización del domicilio, el tamaño del hogar, la presencia de menores en el hogar y la región. Las autoras hallaron que el consumo de alimentos fuera del hogar ha aumentado, y que el consumo de alimentos fuera del hogar es mayor entre los hombres que entre las mujeres, y más frecuente en el área urbana que en la rural, y entre los jóvenes.

En dicho orden de ideas, en línea con la literatura revisada y el contexto colombiano, las hipótesis que guían este texto son:

1. El consumo de alimentos fuera del hogar se incrementó en el periodo analizado, como se señaló en la revisión del contexto y en similitud con la literatura estudiada. Además, de acuerdo con Euromonitor International (2018b, 2018c y 2018d), hubo un aumento de los servicios alimentarios en Colombia. Por lo tanto, se espera un incremento porque se supone que los hogares adecuan sus usos en diálogo también con el mercado.

2. El consumo de alimentos aumenta en las zonas urbanas respecto a las zonas rurales; en los hogares de altos niveles educativos respecto a los niveles educativos más bajos; con altas condiciones de vida respecto a condiciones más bajas, y sin presencia de mujeres en el hogar, en hogares conformados principalmente por hombres. Esto también se espera en el tiempo. Por años, la estimación de diferentes modelos corresponde a probar las diferencias de los coeficientes. Esto se puede entender como el test de Chow. ${ }^{9}$

9. Se sugiere al lector revisar la literatura presentada en la sección de referencias. 
La hipótesis 2 se espera en esas direcciones porque sería en las zonas urbanas donde existirían mayores posibilidades de dichos tipos de consumo; porque a mayores niveles educativos se esperaría que hubiese mayores deseos que probar las diferentes experiencias de consumos alimentarios fuera del hogar; porque a niveles mayores de condiciones de vida se esperaría que las personas pudieran consumir un servicio que está asociado más al ocio. Además, porque los hombres estarían, primero, menos asociados a actividades que implican trabajo en el hogar (como podría ser la preparación de alimentos en casa); segundo, porque los hombres pueden acceder mayoritariamente a bienes y servicios comerciales por la posibilidad de contar con ingresos propios; tercero, porque, en términos de trabajo, al ser los que más cuentan con posibilidades de trabajos formales, tendrían mayores incentivos para usar este tipo de consumo, al estar más tiempo fuera del hogar, lo que quizá también sería una facilidad para suplir una necesidad vital. Se considera que esto se sostiene en el tiempo, porque se parte del supuesto de que las condiciones de desigualdad en Colombia, en términos sociales y económicos (educación, condiciones de vida, mujer, etcétera), se mantienen en el tiempo.

De modo que este trabajo, bajo el entramado teórico presentado, el caso escogido y la metodología a emplear, no había sido desarrollado, por lo que se espera que sea una aportación al estudio del consumo de alimentos en los hogares en diferentes latitudes.

\section{Datos y metodología}

\subsection{La encuesta}

Se utiliza la Encuesta Nacional de Calidad de Vida (ENCV), realizada en Colombia por el Departamento Nacional de Estadística (DANE), para los años 1993, 1997, 2010 y 2014. La elección de los años tuvo como propósito identificar patrones colombianos de la proporción del gasto en alimentos fuera del hogar. La encuesta es de cobertura nacional y aporta información sobre las condiciones de vida de los hogares, sus ingresos y gastos totales. Los resultados se han expandido con el fin de producir estimaciones válidas del país.

La ENCV se compone de diferentes módulos. El módulo de gasto tiene rubros semanales, mensuales, trimestrales y anuales. En los rubros semanales —además del gasto en consumo de alimentos fuera del hogar-, se identifican alimentos (carnes: pollo, cerdo, pescado; granos y verduras: fríjoles, arvejas, etcétera; leche y derivados: queso, yogurt; frutas: banano, guayaba; alimentos procesados, entre otros).

La información de las encuestas permite operacionalizar las condiciones socioeconómicas — conjunto de características sociales, financieras y materiales circunstanciales, que pueden ser asumidas como nivel educativo, ocupación, ingreso, tenencia de casa, entre otros (Galobardes et al., 2006a; 2006b) - de diferentes maneras con el módulo de condiciones de vida de los hogares, datos de la vivienda y niveles educativos alcanzados por los integrantes del hogar. De 
acuerdo con los documentos de la Encuesta Nacional de Calidad de Vida, el hogar está definido como persona o conjunto de personas que se asocian para compartir el alojamiento y la alimentación.

\subsection{Las variables independientes}

Las variables independientes vienen de la revisión de la literatura que se ha enfocado al análisis del consumo de alimentos fuera del hogar. Estas variables son la educación, las condiciones materiales, la presencia de al menos una mujer en el hogar, la edad del jefe del hogar, el lugar de origen (urbano, rural, región), entre otras.

Así pues, en línea con lo anterior, el módulo de educación contiene información del nivel educativo máximo alcanzado por cada miembro del hogar y su sexo, entre otros. Con este módulo se calculó: 1. el nivel educativo máximo alcanzado por cada miembro del hogar y se creó una variable dicotómica (superior o más vs. menos de eso); 2. la cantidad de personas totales por hogar; 3. la presencia de al menos una mujer en el hogar, respecto al total de los integrantes del hogar.

Del módulo de datos de la vivienda, se recodificó la variable del material del piso del hogar. Se supone que el material de piso tierra (1) es la categoría que representa hogares con bajas condiciones materiales o condiciones de vida; el de cemento y gravilla (2) es el segundo; el de madera burda es el tercero (3), y el código cuatro corresponde a materiales de piso más sofisticados (baldosa, mármol, madera pulida y alfombra) y representa a los hogares con mayores niveles de ingreso. Esta variable es un proxy de las condiciones materiales y la calidad de vida de los hogares.

Mediante la variable educativa, se supone que existe un acercamiento a discursos o conocimientos sobre los alimentos, sus funciones y tradiciones. Esta variable, junto con la del material del piso, es reveladora de la existencia de ciertas características socioeconómicas no materiales y materiales en el interior del hogar (Bourdieu, 1998; Grignon y Grignon, 1980).

A su vez, la presencia de mujeres en el hogar afecta el comportamiento del gasto en alimentos en el hogar (Gojard et al., 2017; Martens, 1997; Wood, 1992), porque en la mayoría de los casos son las que lideran las acciones de cuidado del hogar y sus integrantes, con la alimentación como una de las principales actividades. Por tal razón, se construyó la variable dicotómica mujer, en el caso de la presencia de al menos una en el hogar.

A su vez, la edad es un indicador de la asociación del ciclo de vida en el gasto para el consumo de alimentos. Para este caso, se tomó la edad reportada por el jefe del hogar en la encuesta. El jefe del hogar es señalado por los hogares. En Colombia el jefe del hogar es la persona que toma decisiones o que aporta el sustento económico del hogar. En ese sentido, identificar la relación de la edad con la proporción del gasto es también importante para poder dilucidar las características socioeconómicas de los hogares, que tienen incidencias en las proporciones del gasto en alimentos para el consumo fuera del hogar. 
A su vez, la cantidad de personas en el hogar y el sector al que este pertenece (rural o urbano) son proxys de su tamaño y contexto. De acuerdo con la literatura, estas variables afectan las cantidades de compra y lo efectivamente adquirido por condiciones de acceso y disponibilidad (Nogueira Bezerra y Sichieri, 2010).

Dadas las particularidades regionales (Grignon, 1986; Nogueira Bezerra y Sichieri, 2010), en niveles de ingresos y estructuras físicas o geográficas, también se construyeron conglomerados y se creó la variable región, a partir de la división político-administrativa regional del territorio colombiano. Las regiones para el análisis son seis: Bogotá Distrito Capital (D. C.), Central (Antioquia, Boyacá, Cundinamarca, Huila, Norte de Santander, Quindío, Risaralda, Santander, Tolima, Caldas), Pacífico (Chocó, Valle, Cauca, Nariño), Caribe (Atlántico, Bolívar, Cesar, Córdoba, La Guajira, Magdalena, San Andrés y Providencia, Sucre), Orinoquía (Meta, Vichada, Casanare, Arauca) y Amazonía (Putumayo, Amazonas, Caquetá, Guaviare, Guainía, Vaupés) (figura 2). La figura 2 tiene como propósito ilustrar la ubicación de Colombia, su capital y la configuración de las regiones.

Como variable de control, es importante resaltar que las regiones en Colombia son altamente disímiles. Hay regiones que son altamente turísticas, como la Caribe. Hay otra que concentra una gran parte del territorio nacional, con departamentos relativamente ricos en comparación con otros, como la región Central. Mientras que hay zonas que son un poco más pobres, como las regiones Pacífica y Amazonía. Además, están las regiones colindan con diferentes países

Figura 2. Mapa de Colombia

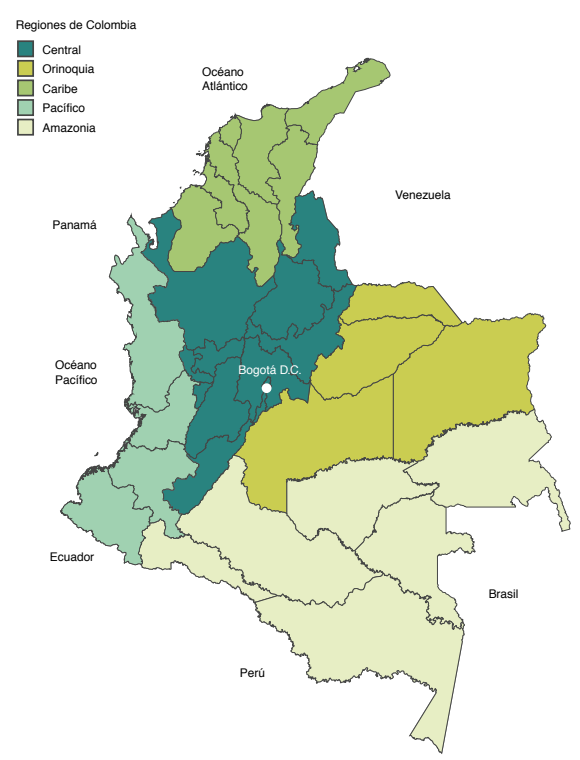


(Ecuador, Perú, Brasil, Venezuela o Panamá) u océanos, lo que puede incidir en la práctica, los productos y los servicios alimenticios.

\subsection{La variable dependiente y el modelo}

Para la construcción de la variable dependiente, primero se revisó el periodo que reporta la encuesta, esto es, si es mensual, semanal o diaria. Se identificó que era semanal. En segundo lugar, se identificaron todas las variables de la encuesta que contenían datos para alimentos, incluida la del consumo de alimentos fuera del hogar. En tercer lugar, dado que la información era semanal, las variables identificadas en alimentos se multiplicaron por cuatro, para hallar un promedio mensual de gasto en alimentos. En cuarto lugar, a partir de las variables identificadas multiplicadas por cuatro, se sumaron todos los gastos en alimentos. Esto daría como resultado el gasto promedio total mensual en alimentos para el hogar. En quinto lugar, se dividió (se halló la proporción) la categoría consumo de alimentos fuera del hogar entre la suma de los gastos en alimentos. Finalmente, en sexto lugar, este resultado se multiplicó por 100, y así se obtuvo la proporción en porcentaje.

Luego, la variable dependiente es la proporción del gasto promedio mensual en alimentos fuera del hogar (G.CFH) respecto al gasto promedio mensual en alimentos del hogar (G.TotAli) para cada hogar. El modelo tomó la siguiente forma:

$\frac{\text { G.CFH }_{i}}{\text { G.TotAli }_{i}}=\beta_{0}+\beta_{1}$ Muj $_{i}+\beta_{2} \operatorname{Edad}_{i}+\beta_{4} \operatorname{NivEdMx}_{i}+\beta_{3}$ MatPiso $_{i}+\beta_{5}$ QPers $_{i}+\beta_{6} \operatorname{Sec}_{i}+\beta_{7} \operatorname{Reg}_{i}+\varepsilon(1)$

Donde el modelo saturado incorpora los años de esta investigación (categórica: 1993, 1997, 2010, 2014) y los modelos no saturados se realizaron para cada año. En resumen, en el ámbito de los hogares ( $i$ ), las variables independientes son las siguientes: presencia de al menos una mujer en el hogar (dicotómica); edad del jefe del hogar (continua); nivel educacional máximo del hogar (dicotómica); material del piso (categórica: tierra, cemento, madera pulida y mármol o cerámica); cantidad de personas en el hogar (continua); sector de la vivienda del hogar (dicotómica: urbana, rural), y región por conglomerados (Central, Pacífico, Caribe, Orinoquía, Amazonía y Bogotá D. C.). La variable dependiente es la proporción del gasto promedio mensual en consumo de alimentos fuera del hogar, reescalada a porcentaje.

Finalmente, sobre la variable épsilon de la ecuación $(\varepsilon)$, por notación, se entiende que los modelos estimados tienen un componente de error que implica que las variables observadas pueden diferir de la curva estimada, porque se reconoce que hay componentes que inciden en la variable dependiente que no son explicados por las variables estimadas en el modelo, lo que induciría al sesgo, razón por la cual sería preciso revisar la bondad de ajuste (R cuadrado). 
Tabla 1. Características de las variables del modelo de regresión lineal, Colombia, 1993-2014

\begin{tabular}{|c|c|c|c|c|c|c|c|c|c|}
\hline \multirow[b]{3}{*}{ Variable } & \multirow[b]{3}{*}{ Categoría } & \multicolumn{8}{|c|}{ Año } \\
\hline & & \multicolumn{2}{|c|}{1993} & \multicolumn{2}{|c|}{1997} & \multicolumn{2}{|c|}{2010} & \multicolumn{2}{|c|}{2014} \\
\hline & & $\mathrm{N}$ & $\%$ & $\mathrm{~N}$ & $\%$ & $\mathrm{~N}$ & $\%$ & $\mathrm{~N}$ & $\%$ \\
\hline \multirow{3}{*}{$\begin{array}{l}\text { Presencia de al } \\
\text { menos una mujer } \\
\text { en el hogar }\end{array}$} & No & 786 & 3,16 & 2.024 & 22,62 & 1.181 & 7,98 & 1.821 & 9,04 \\
\hline & $\mathrm{Si}$ & 24.082 & 96,84 & 6.925 & 77,38 & 13.620 & 92,02 & 18.320 & 90,96 \\
\hline & Total & 24.868 & 100 & 8.949 & 100 & 14.801 & 100 & 20.141 & 100 \\
\hline \multirow[t]{5}{*}{ Edad } & $13-29$ & 3.325 & 13,37 & 579 & 6,47 & 1.794 & 12,12 & 2.298 & 11,41 \\
\hline & $30-44$ & 9.728 & 39,12 & 1.635 & 18,27 & 4.838 & 32,69 & 5.748 & 28,54 \\
\hline & $45-59$ & 6.427 & 25,84 & 1.295 & 14,47 & 4.627 & 31,26 & 6.519 & 32,37 \\
\hline & 600 más & 5.388 & 21,67 & 5.440 & 60,79 & 3.542 & 23,93 & 5.576 & 27,68 \\
\hline & Total & 24.868 & 100 & 8.949 & 100 & 14.801 & 100 & 20.141 & 100 \\
\hline \multirow[t]{5}{*}{ Educación } & Ninguno & 3.533 & 14,86 & 666 & 8,24 & 1.482 & 10,39 & 669 & 3,37 \\
\hline & Bajo & 2.421 & 10,18 & 3.657 & 45,23 & 6.483 & 45,47 & 5.364 & 27,03 \\
\hline & Medio & 11.538 & 48,52 & 3.217 & 39,78 & 5.161 & 36,19 & 11.313 & 57,00 \\
\hline & Alto & 6.286 & 26,44 & 546 & 6,75 & 1.133 & 7,95 & 2.502 & 12,61 \\
\hline & Total & 23.778 & 100 & 8.086 & 100 & 14.259 & 100 & 19.848 & 100 \\
\hline \multirow{5}{*}{$\begin{array}{l}\text { Material del piso } \\
\text { de la vivienda }\end{array}$} & Tierra & 1.223 & 5,35 & 230 & 2,58 & 1.142 & 8,00 & 1.376 & 6,98 \\
\hline & Cemento & 7.308 & 31,97 & 2.723 & 30,59 & 6.215 & 43,56 & 7.675 & 38,94 \\
\hline & Madera & 982 & 4,30 & 683 & 7,67 & 627 & 4,39 & 859 & 4,36 \\
\hline & Mármol & 13.349 & 58,39 & 5.267 & 59,16 & 6.284 & 44,04 & 9.800 & 49,72 \\
\hline & Total & 22.862 & 100 & 8.903 & 100 & 14.268 & 100 & 19.710 & 100 \\
\hline \multirow[t]{3}{*}{ Sector } & Rural & 1.666 & 6,94 & 3.702 & 41,37 & 5.713 & 40,04 & 7.238 & 38,38 \\
\hline & Urbano & 22.327 & 93,06 & 5.247 & 59 & 8.555 & 59,96 & 11.623 & 61,62 \\
\hline & Total & 23.993 & 100 & 8.949 & 100 & 14.268 & 100 & 18.861 & 100 \\
\hline \multirow[t]{7}{*}{ Región } & Central & 8.930 & 37,22 & 3.017 & 33,71 & 3.980 & 28 & 7.368 & 37,38 \\
\hline & Pacífico & 5.470 & 22,80 & 1.614 & 18,04 & 4.106 & 28,78 & 6.204 & 31,48 \\
\hline & Caribe & 4.742 & 19,76 & 1.737 & 19,41 & 2.542 & 17,82 & 3.361 & 17,05 \\
\hline & Amazonía & 45 & 0,19 & 1.449 & 16,19 & 1.898 & 13,30 & 755 & 3,83 \\
\hline & Orinoquía & 14 & 0,06 & 340 & 3,80 & 610 & 4,28 & 492 & 2,50 \\
\hline & Bogotá D. C. & 4.792 & 19,97 & 792 & 8,85 & 1.132 & 7,93 & 1.530 & 7,76 \\
\hline & Total & 23.993 & 100 & 8.949 & 100 & 14.268 & 100 & 19.710 & 100 \\
\hline \multirow{5}{*}{$\begin{array}{l}\text { Cantidad de } \\
\text { personas en } \\
\text { el hogar }\end{array}$} & Variable continua & 24.868 & 100 & 8.949 & 100 & 14.268 & 100 & 18.861 & 100 \\
\hline & $\begin{array}{l}1993 \\
\text { Mínimo: } 1 \\
\text { Máximo: } 12\end{array}$ & & & & & & & & \\
\hline & $\begin{array}{l}1997 \\
\text { Mínimo: } 1 \\
\text { Máximo: } 13\end{array}$ & & & & & & & & \\
\hline & $\begin{array}{l}2010 \\
\text { Mínimo: } 1 \\
\text { Máximo: } 18\end{array}$ & & & & & & & & \\
\hline & $\begin{array}{l}2014 \\
\text { Mínimo: } 1 \\
\text { Máximo: } 16\end{array}$ & & & & & & & & \\
\hline
\end{tabular}

Fuente: elaboración propia a partir de la ENCV 1993, 1997, 2010 y 2014. 


\section{Resultados}

\subsection{Análisis descriptivo}

En Colombia, el gasto en el consumo de alimentos fuera del hogar aumentó entre 1993 y 2014. Para el año 1993, el promedio de la proporción del gasto en consumo de alimentos fuera del hogar fue del $3,7 \%$, y se incrementó de manera sostenida hasta que en el año 2014 fue del 34,64\%.

A medida que el material del piso se hace más sofisticado, la proporción del gasto en consumo de alimentos fuera del hogar aumenta. Para el año 1993, la menor proporción de gasto en consumo fuera del hogar fue para los hogares cuyo material del piso era tierra $(5,29 \%)$ y la máxima fue para aquellos que tenían como material el cemento (10,55 \%). Para el año 1997, nuevamente la menor proporción de gasto en consumo fuera del hogar fue para los hogares cuyo material del piso era tierra $(11,78 \%)$, pero esta vez la mayor proporción fue para los hogares con material de piso de mármol o cerámica $(29,81 \%)$. Igualmente, en el año 2014, la menor proporción fue para los hogares cuyo material del piso era tierra $(26,87 \%)$ y la mayor para los hogares con material del piso de mármol o cerámica $(37,73 \%)$.

Cuando se examina la variable dependiente por nivel educativo, se encuentra que los hogares con mayores niveles tienen una proporción más alta en consumo de alimentos fuera del hogar, en contraste con los que tienen menores niveles educativos, durante todo el periodo. Sin embargo, los resultados evidencian que la proporción se incrementó en los hogares con menores niveles educativos.

Por sector urbano o rural, en 1993, para las zonas rurales, no se reportaron consumos fuera del hogar, y en las zonas urbanas - si existían- eran muy bajos. En cambio, para los años 1997, 2010 y 2014 se reportan consumos de alimentos fuera del hogar en las zonas rurales, pero menores que en las zonas urbanas. Aunque la proporción rural es menor, vale la pena resaltar que pasó del $0 \%$ en 1993 al 28,15\% en 2014.

Por otra parte, se encuentra que hay una asociación negativa en el gasto para alimentos fuera del hogar cuando hay al menos una mujer en el hogar. Adicionalmente, por cantidad de personas en el hogar, se percibe una relación inversa, ya que, a medida que aumenta la cantidad de personas en el hogar, disminuye la proporción del gasto destinado al consumo fuera del hogar.

Cuando se revisa la proporción por regiones (tabla 2), se encuentra que existen diferencias estadísticamente significativas en la proporción del gasto en consumo de alimentos fuera del hogar según la región. En el año 1993, el promedio más alto fue en Bogotá D. C. (13,52 \%) y el más bajo en la Orinoquía $(1,76 \%)$. En 1997, el más alto también fue en la capital Bogotá D. C. (32,08 \%) y el más bajo en la región de la Amazonía (24,12 \%). En 2010, el más alto fue en la Orinoquía (36,21 \%) y el más bajo en la Central $(25,28 \%)$, y en 2014 el más alto en la Amazonía (38,71 \%) y el más bajo en la Central $(31,63 \%)$. 
Tabla 2. Análisis de la varianza (ANOVA): comparación de medias por conglomerado. Datos en porcentaje y por año

\begin{tabular}{|c|c|c|c|c|c|c|c|c|}
\hline \multirow[b]{3}{*}{ Región } & \multicolumn{8}{|c|}{ Año } \\
\hline & \multicolumn{2}{|c|}{1993} & \multicolumn{2}{|c|}{1997} & \multicolumn{2}{|c|}{2010} & \multicolumn{2}{|c|}{2014} \\
\hline & Promedio & $\begin{array}{c}\text { Desv. } \\
\text { Estándar }\end{array}$ & Promedio & $\begin{array}{c}\text { Desv. } \\
\text { Estándar }\end{array}$ & Promedio & $\begin{array}{c}\text { Desv. } \\
\text { Estándar }\end{array}$ & Promedio & $\begin{array}{c}\text { Desv. } \\
\text { Estándar }\end{array}$ \\
\hline Central & 5,523 & 16,389 & 26,292 & 26,540 & 25,280 & 24,360 & 31,634 & 28,913 \\
\hline Pacífico & 5,366 & 16,593 & 29,713 & 27,686 & 31,214 & 28,108 & 32,792 & 30,241 \\
\hline Caribe & 6,860 & 16,862 & 24,202 & 23,325 & 29,681 & 28,706 & 38,421 & 32,579 \\
\hline Amazonía & 4,938 & 19,125 & 24,120 & 24,933 & 31,158 & 27,260 & 38,715 & 29,328 \\
\hline Orinoquía & 1,768 & 6,615 & 32,036 & 29,007 & 36,217 & 29,970 & 35,764 & 30,206 \\
\hline Bogotá D. C. & 13,526 & 20,225 & 32,084 & 22,541 & 31,653 & 24,202 & 36,674 & 26,687 \\
\hline ANOVA & Prob $>F$ & 0,000 & Prob $>F$ & 0,000 & Prob $>F$ & 0,000 & Prob $>F$ & 0,000 \\
\hline
\end{tabular}

Fuente: elaboración propia a partir de la ENCV 1993, 1997, 2010 y 2014.

\subsection{Modelo de regresión lineal}

La tabla 3 presenta las asociaciones de distintas variables socioeconómicas en la proporción del gasto en consumo de alimentos fuera del hogar. El modelo saturado es el conjunto completo de datos, incluyendo la variable año, mientras que para los otros modelos se emplearon los datos por año. Se trabajaron los datos de esta manera para poder identificar los cambios en las variables independientes. Algunas variables independientes se comportan de manera similar durante todo el periodo y el modelo saturado, pero algunas no siguen el mismo patrón durante el tiempo de análisis de esta investigación y el modelo saturado.

Las variables independientes que siguen un patrón similar en el periodo analizado y el modelo saturado son las siguientes: la presencia de mujeres en el hogar, la cantidad de personas en el hogar y la variable sector (urbano/rural) al que pertenece el hogar. De estas variables, la presencia de al menos una mujer en el hogar arroja una relación negativa en la proporción del gasto. Si existe la presencia de al menos una mujer en el hogar, la proporción de consumo de alimentos fuera del hogar disminuye. Esta asociación negativa es sostenida en todos los años analizados, como en el modelo saturado $(\mathrm{p}<0,01)$, de modo que se puede suponer que el hecho de existir al menos una mujer en el hogar es un factor fuertemente explicativo para comprender el consumo de alimentos fuera del hogar. Es decir, la presencia de mujeres en el hogar hace que disminuya la proporción del gasto en consumo fuera del hogar.

Igualmente, la variable cantidad de personas en el hogar tiene una relación inversa con la variable dependiente, ya que, a medida que aumenta la cantidad de personas en el hogar, la proporción del gasto en el consumo de alimentos fuera del hogar disminuye. Este resultado se obtiene tanto en el modelo saturado como en los modelos de cada año $(\mathrm{p}<0,01)$.

En el modelo saturado y en los modelos por cada año, también se encuentra la existencia de una relación directamente proporcional entre el hecho de que el 
hogar pertenezca a la zona urbana y la proporción del gasto en consumo de alimentos fuera del hogar. La proporción del gasto en alimentos fuera del hogar se incrementa cuando el hogar pertenece a la zona urbana, de modo que el hecho de que el hogar pertenezca a la zona urbana indica que existe una propensión mayor a destinar parte del presupuesto a este tipo de consumos $(\mathrm{p}<0,01)$.

En el modelo saturado, la edad tiene una relación negativa $(\mathrm{p}<0,01)$. A medida que aumenta la edad del jefe del hogar, la proporción del gasto en alimentos fuera del hogar disminuye. No obstante, esta relación no es sostenida en el tiempo. Solo para el año 1993, el signo hallado se mantiene $(\mathrm{p}<0,01)$, pero para el resto de los años los resultados no son estadísticamente significativos.

En el modelo saturado, el nivel educativo tiene una relación directa positiva con la proporción del gasto de alimentos fuera del hogar, lo que permite suponer que los hogares con más altos niveles educativos consumen más de su presupuesto en este tipo de abastecimiento de alimentos $(\mathrm{p}<0,01)$. Al analizarse esta variable por años, también se encontró que existe un patrón de la relación entre el nivel educativo y la proporción del gasto en alimentos fuera del hogar. Así, cuando el nivel educativo máximo alcanzado del hogar es superior o más, la proporción del gasto en consumo de alimentos fuera del hogar se incrementa $(\mathrm{p}<0,01 \mathrm{y} \mathrm{p}<0,05)$.

La variable material del piso en el hogar, como proxy de las condiciones materiales, en el modelo saturado no arroja un resultado estadísticamente significativo. Esta variable solo es estadísticamente significativa para 1993 $(\mathrm{p}<0,01)$, año en el que se puede afirmar que existen evidencias estadísticas para creer que el consumo de alimentos fuera del hogar se daba en los hogares con mayores condiciones de vida, ya que, a medida que el material del piso se hace más sofisticado (pasa de material tierra a mármol), la proporción aumenta. De todos modos, esto no es posible afirmarlo para el resto de los años.

Según el aspecto geográfico, teniendo como categoría de referencia la región Central (Antioquia, Boyacá, Cundinamarca, Huila, Norte de Santander, Quindío, Risaralda, Santander, Tolima, Caldas) respecto a las otras regiones, se encuentra que hay una asociación positiva en la proporción cuando no se pertenece a la región Central: Pacífico (Chocó, Valle, Cauca, Nariño) $(\mathrm{p}<0,05)$, Caribe (Atlántico, Bolívar, Cesar, Córdoba, La Guajira, Magdalena, San Andrés y Providencia, Sucre) ( $\mathrm{p}<0,01)$, Orinoquía (Meta, Vichada, Casanare, Arauca) ( $<<0,01)$, Amazonía (Amazonas, Caquetá, Guainía, Guaviare, Putumayo, Vaupés) $(\mathrm{p}<0,01)$ y Bogotá $\mathrm{D}$. C. $(\mathrm{p}<0,1)$. La asociación regional también evidencia que el hecho de estar en la capital de Colombia (Bogotá D. C.) indica una mayor proporción en el gasto para consumo fuera del hogar para casi todos los años de análisis $(\mathrm{p}<0,01)$, incluso teniéndose como categoría de referencia Región Central, una de las regiones más importantes y ricas de Colombia. Finalmente, en perspectiva temporal, tomando como categoría de referencia el año 1993, a medida que pasó el tiempo, la proporción del gasto en alimentos fuera del hogar aumentó $(\mathrm{p}<0,01)$. Se le señala al lector que las estimaciones están ajustadas por el diseño muestral y la heteroscedasticidad. 
Tabla 3. Modelos de regresión lineal. Variable dependiente: proporción de gasto en consumo de alimentos por fuera del hogar. Variables independientes: caractertísticas socioeconómicas de los hogares. Modelo saturado y por años.

\begin{tabular}{|c|c|c|c|c|c|}
\hline & (1) & (2) & (3) & (4) & (5) \\
\hline Modelo Variables & Modelo saturado & 1993 & 1997 & 2010 & 2014 \\
\hline Mujer $=\mathrm{Si}$ & $-26,63^{\star \star \star}$ & $-32,58^{\star \star \star}$ & $-6,860^{\star \star \star}$ & $-23,17^{\star \star \star}$ & $-24,72^{\star \star \star}$ \\
\hline (Ref. = No) & $(0,798)$ & $(1,727)$ & $(1,608)$ & $(1,782)$ & $(1,438)$ \\
\hline Edad 30-44 años & $-1,549^{\star \star * *}$ & $-1,946^{\star \star \star}$ & $-0,181$ & $-0,941$ & 0,701 \\
\hline (Ref. = 13-29 años) & $(0,416)$ & $(0,440)$ & $(2,605)$ & $(1,570)$ & $(1,379)$ \\
\hline \multirow[t]{2}{*}{ 45-59 años } & $-1,490^{\star \star \star}$ & $-2,125^{\star \star \star}$ & 0,0666 & $-1,702$ & 2,033 \\
\hline & $(0,442)$ & $(0,462)$ & $(2,711)$ & $(1,635)$ & $(1,418)$ \\
\hline \multirow[t]{2}{*}{60 o más años } & $-2,829^{\star \star \star}$ & $-4,421^{\star \star \star}$ & $-3,487$ & $-1,281$ & 1,968 \\
\hline & $(0,458)$ & $(0,470)$ & $(2,428)$ & $(1,881)$ & $(1,562)$ \\
\hline Nivel educativo: Bajo & $-0,712$ & $-0,357$ & 1,157 & 0,411 & $-3,491$ \\
\hline (Ref. = Ninguno) & $(0,517)$ & $(0,541)$ & $(3,023)$ & $(2,623)$ & $(5,131)$ \\
\hline \multirow[t]{2}{*}{ Medio } & $-0,833^{\star \star}$ & $-0,234$ & $-0,961$ & 0,695 & $-4,287$ \\
\hline & $(0,349)$ & $(0,329)$ & $(3,051)$ & $(2,680)$ & $(5,072)$ \\
\hline \multirow[t]{2}{*}{ Alto } & $1,952^{\star \star \star}$ & $1,987^{\star \star \star}$ & 4,597 & 4,425 & $-0,431$ \\
\hline & $(0,394)$ & $(0,377)$ & $(3,350)$ & $(2,860)$ & $(5,133)$ \\
\hline Material del piso $=$ Cemento & $2,074^{\star \star \star}$ & $1,650^{\star \star \star}$ & 2,211 & $-0,680$ & $6,108^{\star \star}$ \\
\hline (Ref. = Tierra) & $(0,598)$ & $(0,579)$ & $(3,859)$ & $(3,334)$ & $(3,003)$ \\
\hline \multirow[t]{2}{*}{ Madera } & $1,731^{\star \star}$ & $2,014^{\star \star}$ & $-2,820$ & $-1,039$ & 5,064 \\
\hline & $(0,839)$ & $(0,878)$ & $(4,044)$ & $(3,854)$ & $(3,680)$ \\
\hline \multirow[t]{2}{*}{ Mármol } & $2,375^{\star \star \star}$ & $2,019^{\star \star \star}$ & 2,340 & 0,307 & $6,548^{\star \star}$ \\
\hline & $(0,595)$ & $(0,576)$ & $(3,973)$ & $(3,405)$ & $(3,010)$ \\
\hline \multirow[t]{2}{*}{ Cantidad de personas } & $-1,148^{\star \star \star}$ & $-0,495^{\star \star \star}$ & $-5,097^{\star \star \star}$ & $-3,244^{\star \star \star}$ & $-3,897^{\star \star \star}$ \\
\hline & $(0,0647)$ & $(0,0636)$ & $(0,526)$ & $(0,320)$ & $(0,305)$ \\
\hline Sector $=$ Urbano & $6,774^{\star \star \star}$ & & $7,333^{\star \star \star}$ & $4,119^{\star \star \star}$ & $6,810^{\star \star \star}$ \\
\hline (Ref. = Rural) & $(0,679)$ & & $(1,488)$ & $(1,440)$ & $(1,150)$ \\
\hline Región Pacífico & 0,375 & $-0,113$ & 2,793 & $5,022^{\star \star \star}$ & $-1,001$ \\
\hline (Ref. = Central) & $(0,295)$ & $(0,279)$ & $(1,798)$ & $(1,401)$ & $(1,040)$ \\
\hline \multirow[t]{2}{*}{ Bogotá D. C. } & $6,668^{\star \star \star}$ & $7,312^{\star \star \star}$ & $4,000^{\star *}$ & $5,074^{\star \star \star}$ & $3,681^{\star \star *}$ \\
\hline & $(0,320)$ & $(0,330)$ & $(1,658)$ & $(1,446)$ & $(1,383)$ \\
\hline Año 1997 & $11,37^{\star \star \star}$ & & & & \\
\hline$($ Ref. $=1993)$ & $(0,681)$ & & & & \\
\hline \multirow[t]{2}{*}{2010} & $18,45^{\star \star \star}$ & & & & \\
\hline & $(0,535)$ & & & & \\
\hline \multirow[t]{2}{*}{2014} & $21,53^{\text {** }}$ & & & & \\
\hline & $(0,493)$ & & & & \\
\hline \multirow[t]{2}{*}{ Constante } & $28,60^{\star \star \star}$ & $39,02^{\star \star \star}$ & $33,29^{\star \star \star}$ & $50,78^{\star \star \star}$ & $52,43^{\star \star \star}$ \\
\hline & $(1,259)$ & $(1,826)$ & $(5,289)$ & $(4,205)$ & $(5,742)$ \\
\hline Observaciones & 28,428 & 20,237 & 1,689 & 2,677 & 3,825 \\
\hline R cuadrado & 0,345 & 0,164 & 0,150 & 0,216 & 0,244 \\
\hline
\end{tabular}

Errores estándar robustos en paréntesis

${ }^{* * *} \mathrm{p}<0,01,{ }^{* *} \mathrm{p}<0,05,{ }^{*} \mathrm{p}<0,1$

Fuente: elaboración propia a partir de la ENCV 1993, 1997, 2010 y 2014. 


\section{Discusión}

Primero, en términos generales, los resultados muestran que la proporción del gasto en consumo de alimentos fuera del hogar se incrementó en Colombia en el periodo analizado. En referencia a las características socioeconómicas de los hogares colombianos, se encontraron evidencias estadísticamente significativas sobre las asociaciones de las variables dependientes con el gasto para consumo de alimentos fuera del hogar, de las variables independientes: presencia de mujeres en los hogares colombianos, sus niveles educativos máximos, sus condiciones de vida, materializados en las condiciones de la vivienda del hogar, como lo es el material del piso. Igualmente, respecto a las características contextuales de los hogares, existen relaciones estadísticamente significativas en la variable dependiente de este análisis, dada la cantidad de personas en el hogar, el sector (urbano/rural) y la región (Central, Caribe, Pacífico, Amazonía, Orinoquía o Bogotá D. C.) donde se ubica el hogar.

Segundo, las hipótesis planteadas han sido comprobadas: el consumo de alimentos fuera del hogar se incrementó en el periodo analizado. El consumo de alimentos aumenta en las zonas urbanas, en los hogares de altos niveles educativos, con altas condiciones de vida y sin presencia de mujeres en el hogar.

Tercero, los resultados evidencian diferentes continuidades y rupturas en las prácticas alimentarias colombianas, específicamente en lo concerniente al consumo de alimentos fuera del hogar. Entre los cambios, se puede decir que, al inicio del periodo de análisis, la proporción del gasto en alimentos para consumo fuera del hogar era baja, e incluso inexistente en las zonas rurales, pero al final del periodo dejó de ser baja e incluso llegó a ser casi un tercio del presupuesto del gasto de los hogares colombianos, y aumentó significativamente en las zonas rurales. Además, este tipo de consumo dejó de ser excepcional para las personas con bajos niveles educacionales o de calidad de vida. Con estos resultados, se puede decir que posiblemente este tipo de consumo de alimentos dejó de ser excepcional tanto para las zonas urbanas como para los hogares con determinadas características socioeconómicas. De este modo, los resultados permiten inferir que consumir alimentos fuera de los hogares colombianos es una práctica alimentaria que se ha extendido en el periodo analizado. Ahora bien, si se ponen en diálogo los resultados con el contexto presentado, este resultado es esperable si se tiene en cuenta que en el país el sector de servicio de alimentos se ha expandido de manera continuada en el periodo analizado, tanto en modalidades formales como informales. Entre las modalidades informales, la comida callejera, como lo son las frituras, las comidas rápidas y similares, es de precios relativamente bajos, lo que hace que los sectores con más bajos niveles educativos o de ingreso accedan a comer fuera del hogar. Sin embargo, durante el periodo de análisis, se mantiene la asociación positiva para los hogares con altos niveles educativos y de condiciones materiales.

Por otra parte, siguiendo con el diálogo de los resultados con el contexto, se puede inferir que los resultados son también el reflejo de múltiples transformaciones socioeconómicas estructurales, agenciales, materiales y no materiales de la práctica alimentaria. El incremento de las proporciones del gasto 
en alimentos para el consumo fuera de los hogares colombianos se alinea con la tesis que aboga por la diversificación y la modernidad alimentaria, junto con la estratificación o la distinción en la práctica (Fischler, 1979; Warde et al., 1999). La diversificación y la modernidad alimentarias, entendidas como cambios en los modos de ejecutar la práctica alimentaria, pueden ser afirmadas desde el incremento en la proporción del gasto de este tipo de consumo, pero igualmente, haciendo uso del contexto alimentario para interpretar los resultados (Díaz-Méndez y García-Espejo, 2017; Maloutas y Fujita, 2012), también se puede inferir la diversificación, dado que en Colombia el sector servicio de alimentos ha cambiado de manera sostenida (Euromonitor International, 2018a; 2018b; 2018c; 2018d).

La diferenciación y la diversificación encontradas con los resultados obtenidos en la sección anterior son relevantes en la práctica alimentaria colombiana (Bourdieu, 1998; Grignon y Grignon, 1980; Fischler, 1979; Warde, 1997). Por una parte, cada categoría socioeconómica de los hogares colombianos escogida para el análisis arroja una relación estadísticamente significativa. El hecho de ser hombre o mujer, tener un bajo o alto nivel educativo o de ingreso, habitar la zona rural o la urbana o pertenecer a determinada región de Colombia tiene una relación distinta con la proporción del gasto en alimentos consumidos fuera del hogar. Sin perder de vista el contexto, pues, existen numerosos cambios en el sistema de consumo colombiano. Pero más aún, cuando se analiza en conjunto la distinción y la diversificación en perspectiva temporal, se puede suponer un tipo de distinción móvil, y el consumo de necesidad o de lujo, de ocio o conspicuo también se hace dinámico (Veblen, 2000).

Los resultados muestran que la distinción no es estática en el tiempo ni en el espacio. Así, de nuevo, al analizar los resultados obtenidos en contexto, se puede inferir que los nuevos nichos de mercado han creado distinciones y diferenciaciones en lo que corresponde a la proporción destinada a este tipo de consumo que se refleja en los datos. Es decir, cada año las asociaciones, o la relación de las categorías socioeconómicas, son diferentes en los gastos del consumo de alimentos fuera del hogar, y —al unísono- el mercado también lo ha sido, ${ }^{10}$ como también las estructuras institucionales del sector $\mathrm{y}$, en general, las condiciones socioeconómicas en el país. Con los resultados obtenidos, se puede suponer que las distinciones, aunque siguen existiendo, lo hacen en otros modos y la diversificación también toma otras formas (Bourdieu, 1998; Lamont, 1992; Mennell, 1987; Ritzer, 2001; Veblen, 2000; Warde et al., 1999).

Entre las continuidades, se evidencia el impacto de la tradición con la presencia de al menos una mujer en el hogar. Es decir, si bien el rol de la mujer ha cambiado (Warde, 1997), por ser ella, al mismo tiempo, proveedora y consumidora, también es cierto que en Colombia estos resultados reflejan que ella no ha perdido su rol como responsable de la práctica alimentaria y,

10. Como se ha tratado de decir, el mercado es otro en estrategias, en productos y en servicios. Las unidades vendidas, los valores de los diferentes negocios y las expectativas de los empresarios son también distintos. 
posiblemente, de las diferentes actividades que la componen (abastecimiento, almacenamiento, preparación, servido, etcétera). Los resultados obtenidos indican que la mujer tiene una relación estadísticamente significativa negativa en la proporción del gasto en alimentos para el consumo fuera del hogar, lo que permite inferir que la presencia de mujeres en el hogar indica una propensión más alta a consumir alimentos dentro del hogar, posiblemente porque es ella quien ejecuta dicha labor, mientras que el hecho de ser hombre - en sentido contrario - hace que se destine una proporción mayor al consumo de alimentos fuera del hogar, quizá por el hecho de que el hombre no se hace cargo de este tipo de actividades.

Otra continuidad está en la distinción urbana/rural en el periodo trabajado. En las zonas urbanas, la proporción del gasto en consumo de alimentos fuera del hogar es mayor. Esto podría explicarse quizá por el hecho de que en las ciudades existen más posibilidades de acceso a este tipo de servicios y mayores niveles de ingreso para consumirlos. Además, la capital de Colombia es la zona que, de manera sostenida en el tiempo, tiene asociaciones estadísticamente significativas positivas en la proporción del gasto en consumo de alimentos fuera del hogar. Esto ocurre, quizá, por la estructura de ciudad, ya que Bogotá D. C. se caracteriza por la difícil circulación vehicular y las amplias distancias a recorrer, lo que puede hacer que la gente se vea más obligada a comer fuera del hogar, cerca de sus lugares de estudio o de trabajo. Además, es una ciudad que cuenta con amplias posibilidades de servicios de alimentos y es la zona del país con más cantidad de personas de niveles educativos y condiciones materiales altos. Así pues, comer fuera del hogar no es solo una posibilidad o limitación que resuelven los hogares dadas sus limitaciones y posibilidades, sino que también esta modalidad de consumo alimentario depende de las condiciones materiales o contextuales donde dicha práctica toma existencia (Edwards, 2013; Harris, 2009). Es un hecho que las condiciones del sistema del sector servicio de alimentos en Colombia son diferentes en cada región, e incluso dentro de sus regiones, en función de si es un sector urbano o rural, y los resultados reflejan estos aspectos contextuales.

Por otra parte, la variable cantidad de personas tiene una relación inversa con la proporción del gasto en consumo de alimentos fuera del hogar, ya que, a medida que aumenta la cantidad de personas en el hogar, la proporción del gasto en este tipo de consumo tenderá a disminuir. Esto podría significar que los hogares de gran tamaño no pueden permitirse o tienen mayores restricciones para ejecutar esta modalidad de consumo.

Así pues, por una parte, el patrón de consumo y el incremento encontrados en los resultados permiten afirmar que la población colombiana está modificando su práctica alimentaria y respondiendo a los cambios estructurales (Fischler, 1979; Grignon y Grignon, 1999; Holm et al., 2012; Holm et al., 2015; Mennell, 1987; Poulain, 2002; Warde, 1997). Al mismo tiempo, los vestigios de la tradición en género, niveles educativos y diferencias rurales o urbanas son fuertes (Warde, 1997), con solo algunos cambios. Esta modalidad de consumo de alimentos ejemplifica los conflictos y consensos entre la tradición y la 
modernidad (Fischler, 1979; Grignon y Grignon, 1999) de lo que configura la práctica alimentaria en bienes, servicios, gustos, posibilidades, entre otros.

Aunque la variable región es de control, vale decir que analizar el consumo de alimentos en Colombia en perspectiva regional es un gran reto. Además de las numerosas transformaciones que ha experimentado el país en aspectos culturales, políticos y económicos durante el periodo de análisis, este cuenta con un territorio amplio, con regiones altamente heterogéneas, no solo en materia geográfica, sino también en aspectos culturales y prácticas alimenticias. El acceso $^{11}$ a los alimentos, los productos obtenidos y las preparaciones no son iguales en el vasto territorio nacional. Las tradiciones y preferencias culinarias difieren entre una región y otra, entre un sector y otro.

De este modo, un hogar prototipo del consumo de alimentos fuera de él estaría compuesto por hombres, por una baja cantidad de personas, de altos niveles educativos y de condiciones materiales, perteneciente a las zonas urbanas, especialmente de la capital. Estos resultados se corresponden con la literatura, aunque es un caso latinoamericano y un contexto diferente a los ya estudiados, lo que revela la existencia de un patrón de ejecución de la práctica alimentaria en diferentes latitudes (Nogueira Bezerra y Sichieri, 2010; Díaz-Méndez y García-Espejo, 2017; Díaz-Méndez y Brock, 2017; García Arancibia et al., 2013; Llamas et al., 2012; Lund et al., 2017; Nogueira et al., 2013; Paddock et al., 2017; Martens, 1997; Warde y Martens, 2003; Wood, 1992). En términos sociológicos, y en especial en la sociología del consumo de alimentos, este resultado es importante porque nos invita a seguir indagando en la diferenciación social y económica que se puede percibir a través del consumo de alimentos. Sabemos que cada país latinoamericano o europeo tiene sus propias particularidades alimentarias, y que existen diferencias en productos y servicios alimentarios. Al mismo tiempo, bajo diferentes metodologías y diferentes casos de estudio, percibimos similares patrones de asociaciones, o relaciones, de la variable dependiente (consumo fuera del hogar) con las variables independientes que se trabajaron en este artículo. Así, surgen las siguientes preguntas: ¿en qué nivel contextual (micro, meso, macro) podemos hablar de diferenciación social o económica que pueda ser percibida por medio del consumo de alimentos?, ‘a qué variables podemos dirigirnos y bajo qué metodologías podemos hallar y explicar esas diferencias entre países, que sabemos que existen?, ¿cómo podemos seguir aproximándonos a nuestra compleja realidad?

\section{Conclusiones}

Como categoría analítica teórica y empírica, el consumo de alimentos fuera del hogar permite el acercamiento a la realidad de un lugar y en un tiempo específico en su más vasta definición: económica, cultural, geográfica, etcétera.

11. Por cuestiones de orden público, políticas públicas de salubridad alimentaria, la oferta de bienes o servicios, la infraestructura rural o citadina, etcétera. 
Comer fuera del hogar es una práctica alimentaria que requiere múltiples y diversos elementos para ser ejecutada. Por una parte, hay elementos socioeconómicos agenciales, materiales y no materiales; por otra, hay variables estructurales, materiales y no materiales, que determinan y moldean el consumo de alimentos fuera del hogar.

Ahora bien, aunque con los datos disponibles hasta hoy no es posible llegar a un análisis tan complejo de la categoría que aquí se propuso estudiar - consumo de alimentos fuera del hogar-, con los datos que poseemos para esta investigación -la proporción del gasto en alimentos fuera del hogar y las variables independientes escogidas para el análisis (presencia de una mujer en el hogar, edad del jefe del hogar, nivel educativo, nivel de calidad de vida, cantidad de personas, sector y región)—, se devela un prototipo de consumidor, con su respectivo contexto temporal y espacial. Al hacerse metodología cuantitativa, es absolutamente necesario no perder de vista el contexto para poder hacer inferencias explicativas de lo que los datos muestran. Eso fue lo que se trató de hacer en esta investigación.

Este texto buscó ser una aportación al análisis del patrón del gasto en consumo de alimentos fuera del hogar en un país latinoamericano. Este trabajo no se había hecho hasta el momento bajo esta metodología, ni en esta temporalidad, ni en Colombia, ni en América Latina. Por lo tanto, busca ser una aportación tanto en el caso estudiado como en la temporalidad y en la metodología empleada. Aunque son perceptibles los cambios en nuestra vida cotidiana, los resultados obtenidos permiten tener pruebas científicas sobre los cambios y las continuidades en las prácticas alimenticias en diferentes latitudes.

Específicamente en Colombia, el consumo de alimentos fuera de los hogares presenta continuidades y rupturas. Por una parte, en las continuidades, está el rol de la mujer y lo que podría ser su responsabilidad en la práctica alimentaria en diferentes partes del mundo. Aunque este resultado se puede intuir, los resultados obtenidos lo confirman científicamente. Sin embargo, llama la atención que, a pesar de la inclusión de la mujer en el mercado laboral y en el sistema educativo, ella siga estando relacionada fuertemente con este tipo de actividades, cuando quizá era esperable que esta situación cambiara.

Por otra parte, entre las rupturas, se halló el incremento de este tipo de consumo alimentario en los hogares en el periodo analizado, lo que es también una tendencia global y permite inferir una diversificación en el consumo alimentario. Más aún, con ayuda de otras fuentes que permitieron la comprensión de lo que subyace en los resultados obtenidos, se puede inferir una diversificación en los productos y servicios alimentarios que hoy es posible encontrar en el país, en línea con las teorías de la modernidad alimentaria.

Seguir ahondando en las particularidades del consumo de alimentos, también desde otras metodologías, es una tarea que permitirá comprender más a Colombia, u otras sociedades, sus continuidades, sus rupturas, sus paradojas, su población y sus estructuras materiales o no materiales, de niveles micro o macro, dados un tiempo y un lugar específicos. 


\section{Agradecimientos}

Quiero mostrar mis agradecimientos al Centre Maurice Halbwachs (Centre National de la Recherche Scientifique, École Normale Supérieure, École des Hautes Études en Sciences Sociales), donde tuve la oportunidad de realizar mis estancias de investigación, especialmente a las investigadoras Séverine Gojard, Marie Plessz y Anne Lhuissier, por los textos y los diálogos compartidos. También expreso mi gratitud a Luis Maldonado, del Instituto de Sociología de la Pontificia Universidad Católica de Chile, y a los evaluadores dobles ciegos por las sugerencias constructivas que contribuyeron a mejorar la versión final de este artículo. Igualmente, quiero agradecer a la Universidad Santiago de Cali por motivar e incentivar la investigación.

\section{Financiación}

La realización fue posible gracias a la Comisión Nacional de Investigación Científica y Tecnológica de Chile, CONICYT-PCHA/Beca Doctorado Nacional/2017-21170258, y al Instituto de Sociología de la Pontificia Universidad Católica de Chile.

\section{Referencias bibliográficas}

Bourdieu, Pierre (1998). La distinción. Madrid: Grupo Santillana de Ediciones.

Cabiedes-MiragaYa, Laura (2017). «Analysis of the economic structure of the eatingout sector: The case of Spain». Appetite, 119, 64-76. <https://doi.org/10.1016/j.appet.2017.01.018>

Desjeux, Dominique (2006). La consommation. París: Presses Universitaires de France, col. "Que sais-je?».

DíAz-MÉndez, Cecilia y Brock, Hans-Peter van den (2017). «Eating out in modern societies: An overview of a heterogeneous habit». Appetite, 119, 1-4. $<$ https://doi.org/10.1016/j.appet.2017.05.003>

Díaz-Méndez, Cecilia y García-Espejo, Isabel (2017). «Eating out in Spain: Motivations, sociability and consumer contexts». Appetite, 119 (1), 14-22. $<$ https://doi.org/10.1016/j.appet.2017.03.047>

Dubuisson-Quellier, Sophie (2016). «De la routine à la déliberation. Les arbitrages des consommateurs en situation d'achat». Reseaux, 135-136, 253-284. $<$ https://doi.org/10.3917/res.135.0253>

Dubuisson-Quellier, Sophie y Plessz, Marie (2013). «La théorie des pratiques. Quels apports pour l'étude sociologique de la consummation?». Sociologie, 4(4), 451-469. <https://www.cairn.info/revue-sociologie-2013-4-page-451.htm>

EDWARDS, John (2013). «The foodservice industry: Eating out is more than just a meal». Food, Quality and Preference, 27, 223-229. <https://doi.org/10.1016/j.foodqual.2012.02.003>

Eusas, Norbert (2016). El proceso de la civilización. Investigaciones sociogenéticas y psicogenéticas. Traducción de Ramón García Cotarelo. México: Fondo de Cultura Económica.

EUROMONitOR InTERNATIONAL (2015). A new era of growth and competition: global consumer foodservice in 2015 and beyond [en línea]. <www.euromonitor.com> [Último acceso: 20 de abril de 2018]. 
- (2018a). Consumer foodservice global industry overview [en línea]. <www.euromonitor.com> [Último acceso: 20 de abril de 2018].

- (2018b). Consumer foodservice in Colombia: country report [en línea]. <www.euromonitor.com> [Último acceso: 20 de abril de 2018].

- (2018c). Street stalls/kiosks in Colombia [en línea]. <www.euromonitor.com> [Último acceso: 20 de abril de 2018].

- (2018d). Consumer foodservice by location in Colombia: country report [en línea]. <www.euromonitor.com> [Último acceso: 20 de abril de 2018].

FisChler, C. (1979). "Gastro-nomie et gastro-anomie». Communications: La nourriture. Pour une Anthropologie Bioculturelle de l'Alimentation, 31, 189-210. <https://doi.org/10.3406/comm.1979.1477>

Galobardes, Bruna; Shaw, Mary; Lawlor, Debbie; Lynch, John y Smith, George Davey (2006a). «Indicators of socioeconomic position (Part 1)». Journal Epidemiology and Community Health, 60, 7-12. <https://doi.org/10.1136/jech.2004.023531>

- (2006b). «Indicators of socioeconomic position (Part 2)». Journal of Epidemiology and Community Health, 60, 95-101. <https://doi.org/10.1136/jech.2004.028092>

García Arancibia, Rodrigo; Depetris-Guiguet, Edith y Rossini, Gustavo (2013). «Consumo de alimentos fuera del hogar en Argentina. Relevancia de la composición demográfica y tipología de los hogares». Población y Salud en Mesoamérica, 10 (2), 1-19. <https://doi.org/10.15517/psm.v10i2.8512>

Gojard, Séverine; Plessz, Marie y Régnier, Faustine (2017). «Les femmes et l'alimentation: le rôle des normes alimentaires et corporelles». Instituto National de la Recherche Agronomique: Sciences Sociales, 1-2, 1-7.

Grignon, Claude (1986). "Alimentation et régions». Cahiers de Nutrition et de Diététique, 21 (5), 381-389.

Grignon, Claude y Grignon, Christine (1980). «Styles d'alimentation et goûts populaires». Revue Française de Sociologie, 21 (4), 531-569. <https://doi.org/10.2307/3320833>

- (1999). «Long-Term Trends in Food Consumption: A French Portrait». Food and Foodways, 8, 151-174. <https://doi.org/10.1080/07409710.1999.9962086>

Harris, Marvin (2009). Bueno para comer. Madrid: Alianza Editorial.

Herpin, Nicholas (2004). Sociologie de la consommation. París: La Découverte.

Holm, Lotte; Ekström, Marianne; Gronow, Jukka; KJÆrnes, Unni; Lund, Thomas; MäKelä, Johanna y Niva, Mari (2012). "The modernisation of Nordic eating. Studying changes and stabilities in eating patterns». Anthropology of Food, 7, 2-14. $<$ https://doi.org/10.4000/aof.6997>

Holm, Lotte; Lauridsen, Drude; Gronow, Jukka; Kahma, Nina; KJærnes, Unni; Lund, Thomas; Mäkelä, Johanna y Niva, Mari (2015). "The food we eat in Nordic countries - Some changes between 1997 and 2012». Kostvetenskap Rapportserie, 1, 227-246.

Kocevski, Jordan y Risteski, Michael (2012). «Eating out on vacation». Procedia Social and Behavioral Sciences, 44, 398-405. <https://doi.org/10.1016/j.sbspro.2012.05.044>

Lamont, Michèle (1992). Money, Morals, and Manners: The Culture of the French and the American Upper-Middle Class. Chicago y Londres: The University of Chicago Press. <https://doi.org/10.7208/chicago/9780226922591.001.0001> 
LARMET, Gwenael (2002). «L'organisation des achats alimentaires». Cahiers d'Economie et de Sociologie Rurales, INRA Editions, 63, 51-84.

Le Velly, Ronan (2012). Sociologie du marché. Paris: La Découverte.

Llamas Huitrón, Ignacio; Leija, Humberto Armando y Aboites Manrique, Gilberto (2012). "Gasto en alimentos y bebidas fuera del hogar. El caso de México, 1992 y 2008». Economía: Teoría y Práctica, 37, 177-198. $<$ https://doi.org/10.24275/ETYPUAM/NE/372012/Llamas>

Lund, Thomas; KJÆrnes, Unni y Holm, Lotte (2017). "Eating out in four Nordic countries: National patterns and social stratification». Appetite, 119, 23-33. <https://doi.org/10.1016/j.appet.2017.06.017>

Maloutas, Thomas y Fujita, Kuniko (2012). Residential Segregation in Comparative Perspective Making Sense of Contextual Diversity. Inglaterra: Ashgate.

Martens, Lydia (1997). "Gender and the eating out experience». British Food Journal, 99 (1), 20-26. <https://doi.org/10.1108/00070709710158843>

Mennell, Stephen (1987). Français et anglais à table. Du moyen âge à nos jours. París: Flammarion.

Neirinck, Edmond y Poulain, Jean-Pierre (2001). Historia de la cocina y de los cocineros. Técnicas culinarias y prácticas de mesa en Francia, de la Edad Media a nuestros dias. Barcelona: Zendrera Zariquiey.

Nogueira Bezerra, Ilana y Sichieri, Rosely (2010). "Características e gastos com alimentação fora do domicílio no Brasil». Revista de Saúde Pública, 44(2), 221-229.

<https://doi.org/10.1590/S0034-89102010000200001>

Nogueira Bezerra, Ilana; Moura Souza, Amanda de; Alves Pereira, Rosangela y Sichieri, Rosely (2013). "Consumo de alimentos fora do domicilio no Brasil». Revista Saúde Pública, 47 (1), 200S-2011S. <https://doi.org/10.1590/S0034-89102013000700006>

Perry, Guillermo (2011). Economía y Constitución. En Departamento de Ciencia Política, Facultad de Ciencias Sociales (Editor). Constitución de 1991, 20 años: Logros y pendientes, Bogotá: Universidad de los Andes, 38-39.

Poulain, Jean-Pierre (2002). Sociologies de l'alimentation: Les mangeurs et l'espace social alimentaire. París: Presses Universitaires de France.

PrättäLä, Ritva; Berg, Mari-Anne y Puska, Pekka (1992). «Diminishing or increasing contrasts? Social class variation in finish food consumption patterns, 1979-1990». European Journal of Clinical Nutrition, 46 (4), 279-287. PMID: 1600925.

ReckwitZ, Andreas (2002). "Toward a theory of social practices: A development in culturalist theorizing». European Journal of Social Theory, 5 (2), 243-263. <https://doi.org/10.1177/13684310222225432>

Régnier, Faustine; Lhuissier, Anne y Gojard, Séverine (2006). Sociologie de l'alimentation. París: La Découverte.

Ritzer, George (2001). «Thorstein Veblen in the Age of Hyperconsumption». En: Ritzer, George. Explorations in the Sociology of Consumption: Fast Food, Credit Cards and Casinos. Londres: SAGE Publications.

Veblen, Thorstein (2000). Teoria de la Clase Ociosa. DOI: <www.elaleph.com>.

WARDE, Alan (1997). Consumption, food and taste: Culinary antinomies and commodity culture. Londres: SAGE Publications.

Warde, Alan y Martens, Lydia (2000). Eating out: Social differentiation, consumption and pleasure. Cambridge: Cambridge University Press. 
Warde, Alan; Martens, Lydia y Olsen, Wendy (1999). "Consumption and the problem of variety: Cultural omnivorousness, social distinction and dining out». Sociology, 33 (1), 105-127. <https://doi.org/10.1017/S0038038599000061>

Wood, Roy (1992). "Gender and trends in dining out». Nutrition \& Food Science, $5,18-21$. <https://doi.org/10.1108/EUM0000000000966> 